N. Uskow: Zur Entwickelungsgeschichte der Leber und der Lungen. 219

Visceralsp. $=$ Visceralspalten.

Wand d. duct. Cuv. = Wand des ductus Cuvieri.

Wolff $\mathrm{G}$. = Wolf'scher Gang.

Wolff $K .=$ Wolf'scher Körper.

$\mathrm{x}$ (Fig. 6) = der lateralen Schlussecke des geschlossenen Coeloms.

$\mathrm{x}($ Fig. 31$)=$ hintere zum paarigen Coelom gekehrte Wand des Septum transversum.

$\mathrm{x}$ in Fig. $43=$ (siehe die Erklärung im 'Text).

$\mathrm{x}$ in Fig. 41 = Abspaltungswinkel der Membrana pleuro-pericardiaca vom Diaphragma.

$x-z($ Fig. 7$)=$ Grenzlinie $z$ wischen ventralem und dorsalem Theile der Pericardialhöhle.

(Aus dem anatomischen Institute zu Strassburg i. E.)

\title{
Bemerkungen zur Entwickelungsgeschichte der Leber und der Lungen.
}

\author{
Von
}

Dr. N. Uskow

aus Kronstadt in Russland.

Hierzu Tafel VII u. VIII.)

Bei der Untersuchung über die Entwickelungsgeschichte des Zwerchfells und die Theilung des Coeloms im Allgemeinen mussten wir unser Augenmerk auch auf die Entwickelungsgeschichte der Leber und der Lungen richten, wobei wir auf Erscheinungen gestossen sind, die keineswegs eines allgemeinen Interesses ermangeln. Dass die nachstehende kurze Bemerkung unter besonderem Titel erfolgt, findet seine Erklärung in dem Bestreben, die Darstellung einer schon an sich so complicirten Erscheinung, wie die Entwicklung des Zwerchfells, nicht noch weiter zu erschweren. 
Beim Kaninchenembryo besteht am 9. Tage die Randpartie der fovea cardiaca aus dem Hypoblast und dem Mesoblast; Letzterer weist an der Uebergangsstelle von der Herzwand nach der ventralen Wand der primitiven Pericardialhöhle schon eine bedeutende Verdickung auf. Am 10. Tage wird der Rand der fovea cardiaca zu einer mehr quergestellten Platte nud es dringt in dieselbe von der Rïckfläche aus eine besondere Vertiefung des Hypoblasten ein, welche nach allen Autoren die primitive Anlage der Leber ist, wenigstens des Theiles derselben, weleber aus dem Hypoblasten entsteht. Zu gleicher Zeit nimmt die oben erwähnte Verdickung das Aussehen einer ziemlich grossen und dicken Masse mit höckeriger Oberfäche an. Bei einem $5 \mathrm{~mm}$ langen Embryo (vom 11. Tage) ist die ganze Innenfläehe des Bodens der Pericardialhöhle mit Zotten besäet, was sowohl an Quer-, als an Längssehnitten ersichtlich ist.

Bei den Säugethieren bat N. Lieberkiihn zuerst auf die Bedeutung dieser Zotten aufmerksam gemacht; er sagt: „Aehnliche Zotten, wie die von $\mathrm{Hi}$ s (beim Huhn) abgebildeten (Taf. XI. II. 7) kommen an der Eintrittsstelle der vena omphalo-mesenterica vor. Diese finde ich in einem späteren Stadium verlängert und hohl, und an noch älteren mit der aus dem Kopfdarm hervorgetretenen Leberanlage so im Zusammenhange, dass sie die ersten Blutgefässe dieses Organs darstellen ${ }^{1}$ ).

Kölliker ${ }^{2}$ ) findet beim Kaninchen Zotten an dem von Lieberk $\mathfrak{i} h \mathrm{n}$ angegebenen Orte, ausserdem auf dem Vorhofe und der Rückseite der Aorta und fügt hinzu: Davon, dass die erstgenannten Zotten später die ersten Blutgefässe der Leber bilden, konnte ich Nichts finden, vielmehr habe ich dieselben auch in den ersten Zeiten nach der Bildung der Leber noch gesehen.

$\mathrm{His}^{3}$ ) unterscheidet an den Zotten eine äussere Schicht von dunkleren Zellen und eine innere helle Zone; erstere entwickelt sich aus der Muskelschicht des Herzens und der Parietalhöhlenwand, letztere aus deren bindegewebigen, bezw. endothelialeu Anlagen."

1) N. Lieberkïhn, Sitzungsberichte d. Ges. für. Medic. und Naturwissensch. zu Marburg 1876 A. 1.

2) Entwickelungsgeschichte 1879. paug. 815.

3) Archiv f. dnat. u. Eutwickclungsgesch. 1881. paur. 326 , 
Indem er die unmittelbare Betheiligung der inneren Zottenschicht an der Bildung des Gefässgerüstes der Leber zugiebt, fügt er binzu: „Von inneren Zotten fallen die an der Vorhofsbasis und am Septum transversum sich entwickelnden direct in das Gebiet der Leberanlage und eine Ueberführung ihrer Innenzone in deren Gefässgeriist kann nicht wohl bezweifelt werden, ihre Rindenschicht wird ausserbalb der Leber bleiben."

An unseren Präparaten sehen wir folgendes: Die Zotten auf dem Septum transversum und der Ventralwand des Vorbofs haben eine sehr unregelmässige Form; an der Oberfläche sind sie von Zellen bedeckt, welche die unmittelbare Fortsetzung der die Innenfläche der Primitivpericardialhöhle bedeckenden Zellen, also des Coelomepithels, bilden.

Die Höhlung der Zotte communicirt stellenweise mit der Höhle des Sinus venosus und ist manchmal mit einem sehr dentlichen Endothel bekleidet. Unter den Zotten, zwischen ibnen und der Hypoblastschicht, liegt ein Gewebe vom Character eines lockern Bindegewebes. Der Hypoblast bildet an dieser Stelle, wie gesagt, die Einstülpung der epithelialen Leberanlage. Anfangs kann man Schnitte antreffen, wo die Grenze der epithelialen Zellen des Primitivleberganges sehr scharf ausgoprägt ist, in späteren Stadien aber verändern sich die Erscheinungen: einerseits verweben sich die Zotten untereinander und es entsteht ein Gewebe von ganz schwammigem Character, die die Masse der Zotten bildenden Zellen liegen nun innerhalb dieses Gewebes, werden bedeutend grösser und, wie die Karyolinese-Erscheinungen lehren, gehen sie nicht nur nicht zu Grunde, sonderu proliferiren stark; das lockere Bindegewebe mit seinen schwach sich färbenden Zellen und mit bedeutender Grundsubstanz wird nur an der Ventralseite der betreffenden Körperpartie sichtbar; gleichzeitig sieht man die Zellen des Primitivleberganges sich stark unter vielfachen Karyokinese-Erscheinungen vermehren und in mehrfacher Sehicht sich lagern (Taf. VIII, Fig. 1). Auf diese Weise - wir sprechen von einem etwas späteren Stadium - ist es unmöglich, zu unterscheiden, was zu dem aus der Zotte entstandenen Gewebe und was zu dem aus dem Hypoblast entstandenen gehört. Wir wollen damit nicht sagen, dass die Zellen der Zotten sich mit denen des Hypoblasten untermischen, so dass sie an der Bildung der Drüsenzellen der Leber theilnehmen, um so weniger, als hie und da immerhin cylindrisch geordnete und 
scharf abgegrenzte Haufen von Hypoblastzellen anzutreffen sind; wir sagen nur, dass das Gewebe der Zotten mit seinen oberflichlichen Zellen, resp. den vom Epithelinm des Coeloms stammenden Elementen zweifellos an der Bildung der Leber theilnimmt, indem diese Zellen sich in einen Bestandtseil dieses so complicirten Organs verwandeln. In Betreff der Gefüsse der Zotten ist nach dern Gesagten klar, dass unsere Präparate die Meinung der oben citirten Autoren nur bestätigen und somit den fast cavernösen Bau der eben sich entwickelnden Leber vollständig erklären. In späteren Stadien (bei Kaninchenembryoner von $9 \mathrm{~mm}$ ) existiren die Zotten nicht mehr; ob sie nun auf dem beschriebenen Wege, $d$. h. in die Leber ibbergehend, verschwinden, oder ob noch auf andere Weise, können wir nicht sagen; die Thatsache ihrer grösseren Verbreitung nach vorne zu längs der Sinuswand spricht für die Möglichkeit auch der zweiten Voraussetzung.

Kölliker hat im 1. Theile seiner „Entwickelungsgeschichte des Menschen etc." (1879) für das Kaninchen eine doppelte Lungenanlage angenommen. Im 2. Bande dagegen (pag. 861) sagt er, er habe im Laufe der Zeit gefunden, dass beim Kaninchenembryo von 10 Tagen „der Vorderdarm dicht hinter dem die Kiemenspalten zeigenden Abschnitte in eine seitlich comprimirte Gestalt übergeht". „An dem ventralen Abschnitte," - fähitt er fort, „den ich die Lungenleiste nennen will, entsteht am 10. Tage die Lunge selbst dadurch, dass der unterste Theil der Leiste sich erweitert und an beiden Seiten sich ausbuchtet." Indem er eine Reilhe schematischer Querschnitte des Vorderdarmes mit der Lungenanlage eines Embryo von 11 Tagen und 6 Stunden vorfiuhrt, zieht er daraus den Schluss: „Diesem rufolge kann auch für das Kaninchen die erste Anlage der Lungen als eine un paare bezeichnet werden; doch habe ich im Gegensatze zu andern Schilderungen zu betonen, dass die Anlage der Luftröhre sicherlich nicht später als die der Lunge, ja, wie ich glauben möchte, eher vor derselben erscheint" (pag. 860).

F. Balfour spricht sich in seinem ,Handbuch der vergl. Embryologie“ (pag. 688) folgendermassen auș: „Phylogenetisch be- 
trachtet, scheint die Ontogenie der Lunge anzudenten, dass dieselbe zuerst ein unpaariges Gebilde war und erst secundür paarig wurde, uud dass die Trachea erst verhältnissmässig spät auftrat."

Was den Antbeil des Mesoblasten an dem Bildungsprocesse der Lungen und der Trachea betrifft, so sagt $\mathrm{K}$ ölliker nur, dass „Speiseröhre und Lungenanlage in diesem Stadium (am 10. Tage) vorn und seitlich von einer dicken Lage Mesoderma (Darmfaserplatte) umhüllt" seien. Balfour aber behauptet: „Während diese Veründernngen mit der Hypoblastwandung des Darmrohrs vor sich grehen, verdickt sich das diese Gebilde umgebende splanchnische Mesoblast bedentend, zeigt aber sonst keine Spur der in seinem Innern ablaufenden Vorgänge, weshalb die beschriebene Ausbildung der Lungen und der Luftröhre (sie ist bei ihm mit derjenigen in Köllikers Darstellung identisch) von aussen nicht zu beobachten ist" (pag. 687).

Wir besitzen eine lückenlose Serie von Querschnitten eines etwas weniger als 10 Tage alten Kaninchens und lassen hier aus denselben die Contouren des Vorderdarms, wie sie mit Hülfe der camera lucida gezeichnet sind, folgen: s. Fig. 62-86 Taf. VII. (Die Nummern bedeuten die Zahlfolge der Schnitte vom Kopfende an gerechnet.) Am Schnitt 62 wird der Vorderdarm bedeutend schmaler in frontaler Richtung, als vorher, während er seinen frühern Sagittaldurchmesser beibehält. Mit $\mathrm{Nr}$. 66 beginnt auch Letzterer sich zu vergrössern. In Nr. 70 erreichen beide Durchmesser die gleiche Ausdehnung, worauf weiterhin die Ausdebnung in sagittaler Richtung überwiegt und so schnelle Fortschritte macht, dass auf Schnitt 77 das Lumen des Vorderdarms nur noch einen sagittalen Spalt vorstellt. Dabei lebrt uns schon das blosse Augenmaass, dass vom 73. Schnitte an der Sagittaldurehmesser sich eigentlich nicht vergrössert; er bleibit sich vollständig gleich, und die spaltförmige Gestalt des Lumens hat ihre Entstehung ausschliesslich dem Zusamnenrïcken der Seitenwände des Darmes zu verdanken. Auf den Abbildungen ist die Dicke der Vorderdarmwandung angegeben, und es zeigt sich deutlich, dass die Verengerung des Lumens sich aus einer Verdickung derjenigen Hypoblastschicht, welche die genannten Wände bildet, nicht erklären lässt. Dies genügt doch, wie es scheint, zum Nachweise, dass keine Figur aus der vorhergehenden entwickelt werden kann, d. h. die Figuren beweisen zweifellos, dass jeder Abschnitt des Vorderdarmes selbständig entstanden ist, ohne 
in seiner Entwickelung durch die Form der vor ihm gelegenen Schnitte hindurch gegangen zu sein. Auch kann kein Abschnitt durch die Formen der hinter ihm liegenden hindurchgegangen sein, da wir sehen, dass, je mehr nach hinten der Schnitt fällt, um so enger das Lumen des Vorderdarmes ist. Es ist aber ein längst bekanntes Faktum (wovon man sich leicht an jedem Embryo von 9 Tagen iberzeugen kann), dass der Vorderdarm, welcher in dem vorhergehenden Entwickelungsstadium am Kopfende fast den gleichen Durchmesser bewahrt hatte bis zum Vorderrande der fovea cardiaca, bei Letzterem sich rapid erweitert, indem er in den offenen Theil des Hypoblasten libergeht. An Schnitt 77 bemerken wir schon die Theilung des Lumens des Vorderdarmes in einen ventralen und einen dorsalen Abschnitt; dieser letztere ist bedeutend breiter. Nach der Auffassung der Autoren haben wir in dem ventralen Abschnitt die Luftröhranlage, in dem dorsalen die Oesophagusanlage.

In Figg. 81-84 ist das Verhältniss wiederum ungekehrt. Das ventrale Ende zeigt sich bedeutend verbreitert und zwar fast symmetrisch nach beiden Seiten. Diese Verbreiterung ist eben die Lungenanlage.

Fassen wir das Gesagte zusammen, so lïsst sich tiber die Entwicklung der Lungen beim Kaninchen Folgendes aufstellen: 1) Schon der Process des Verschlusses des Vorderdarmes geht in der Weise vor sich, dass in den gesonderten Regionen des Canals von vornherein die Anlage der Theilung in Speiseröhre und Luftröbre gegeben ist. Demnach ist der Process der primitiven Bildung der Luftröbre keineswegs eine Formveränderung des be reits a usge bildeten Vorderdarmes.

2) Die Lungenanlage hat die Gestalt einer unpaaren Ausbuchtung der ventralen Wand des Vorderdarmes.

3) Die Lungenanlage existirt schon, während die Luftrïhre noch die ventrale Abtheilung des Vorderdarmes ausmacht, d. h. während die Luftröhre nur in der ersten Anlage vorhanden ist. Die Anlagen beider Abtheilungen der Athmungsorgane entsteben also gleichzeitig und selbstständig.

4) Die Oeffnung, durch welche beide Abtheilungen des Vorderdarmes in der Region der Lungenanlage communiciren (Figg. $81-83$ ), ist viel schmaler als die Oeffnung, durch welche die Speiseröhrenabtheilung des Vorderdarmes mit der Luftröhrenanlage in Verbindung steht, und der Verschluss tritt an ersterer Stelle 
friiher ein. Mithin erfolgt die Absonderung der Lunge von dem Vorderdarme fruher als die der Luftröhre. Da wir nun den AusŁruck "Anlage" der betreffenden Bildungen in dem Sinne der primitiven Absonderung derselben nehmen, so haben wir in dem Gesagten eine factische Bestätigung der oben citirten Annahme Balf our's, die sich auf die Erscheinungen der Phylogenie gründete.

5) Die Lungenanlage befindet sich in gleicher Höhe mit der Basis des Sinus venosus, unmittelbar vor der Leberanlage (s. Fig. 81 u. 86), wie dies vor Kurzem $\mathrm{His}^{\mathrm{l}}$ ) nachgewiesen hat.

Das wäre alles, was die primitive Anlage der aus dem Hypoblast entstehenden Lunge betrifft. Aber auch der Mesoblast in der entsprechenden Region bleibt nicht ohne Veränderungen.

Vom 79. Sehnitte an beginnt seine Oberfläche sich bedeutend in die Coelomböhle vorzuschieben. Dieses Vorschieben erreicht in der Höhe der Figur 83 sein Maximum und schwindet alsdann in rapider Weise. Entsprechend dieser Veränderung des äussern Contours erfolgt auch eine Verdickung der Mesoblastschicht, d. h. sie biegt nicht einfach nach aussen um, sondern wuchert in der Richtung ihrer Dicke, wobei ihr innerer Contour bei weitem nicht so scharf ist wie an den mehr vorne liegenden Schnitten. Diese Erscheinung erklärt sich natürlich sehr leicht als das Resultat eines Schnittes einer sphärischen Fläche, indessen eine genauere Betrachtung der Präparate bei bedeutenderer Vergrösserung bietet uns etwas Anderes; s. Taf. VIII Fig. 2 (vom Schnitt 81), welcher den ganzen Durchschnitt der betreffenden Region zur leichteren Orientirung wiedergibt.

Der Mesoblast (Mes.) weist hier 4 und mehr Zellenschichten auf; sein innerer Rand ist nicht scharf begrenzt, an einzelnen Stellen gehen die Zellen in Zügen in der Richtung nach der epithelialen Lungenanlage (L) und lagern sich ganz nahe an dieselbe. Der Reichthum an Zellen in Verein mit den Karyokinese-Erscheinungen weist deutlich auf ein energisches Wuchern des Mesoblasts in dieser Region hin. Wir besitzen eine Serie von Schnitteu von einem Embryo auf ungefähr demselben Entwicklungsstadium, wie das den Abbildungen entsprechende, und aus diesen ist ersichtlich, dass in der genannten Region für die Lungenanlage der Mesoblast eine charakteristische Verbreiterung aufweist, während der Hypoblast des Vorder-

1) $\mathrm{His,} \mathrm{Archiv} \mathrm{f.} \mathrm{Anat.} \mathrm{und} \mathrm{Entwickelungsgeschichte} \mathrm{(1381)} \mathrm{p.} \mathrm{303.} \mathrm{ff.}$ 
darmes nur eine geringe Dicke zeigt ohne Vermehrungserscheinungen der Zellen. Hierin haben wir also einen Hinweis daranf, dass bei der Bildung der Lungenanlage die Veränderungen des Mesoblasten zum Theil wenigstens unabhängig von der Wucherung des Hypoblasten vor sich gehen. Dies alles führt uns zu der Ueberzeugung, dass der Mesoblast bei der Entstehung der Lungenanlage nicht passiv bleibt; er dringt in das zwischen ihm und dem Hypoblasten liegende Bindegewebe ein. Als Lungenanlage ist nicht bloss die bekannte Abtheilung des Vorderdarmes anzusehen, sondern ebenso gut auch die in Rede stehende Mesoblastverdickung sammt dem zwischen ihm und dem Hypoblasten liegenden Bindegewebe.

Es gehen also die Zellen des Mesoblasten (d. h. des ursprïnglichen Coelomepithels) in den Bestand der Lungenanlage über, indem sie nicht bloss auf ihrer Oberfläche bleiben, sondern in die Tiefe vordringen; wir vermuthen, dass sie dort zu den museulösen Bestandtheilen der Lunge sich umgestalten.

\section{Erklärung der Tafeln VII u. VIII.}

Taf. VII. Fortlaufende Serie von Querschnitten des Vorderdarmes eines etwas weniger als 10 Tage alten Kaninchenembryos. Die Numerirung der Schnitte beginnt mit demjenigen, an welchem die Anlage der Augen sichtbar wird. Schnitt 62 zeigt uns den Rest der Anlage der letzten Kiemenspalte, In allen Figuren bedeutet: a. = Aorta, c. $=$ Coelom, v. D. = Vorderdarm. v. D. d. = dorsale Abtheilung des Vorderdarms, v. D. v. = ventrale Abtheilung des Vorderdarms, Oes. = Oesophagus, L. = Lunge.

77-80. Spaltförmiges Lumen des Vorderdarmes, wobei die dorsale Abtheilung den Oesophagus (v. D. d.), die ventrale Abtheilung die Trachea darstellt (v. D. v.).

81. Ganzer Schnitt durch den genannten Kaninchen-Embryo: Vordere Abtheilung der Lungenanlage.

a. = Aorta.

oes. = Oesophagus

L. = Lunge.

c. $=$ Coelom. 
sin. $\mathrm{v} .=$ sinus venosus.

p. p. H. = primitive Pericardial-Höhle.

ch. d. = chorda dorsalis.

med. sp. = medulla spinalis.

82-84. Die dorsale Abtheilung des Vorderdarmes stellt den Oesophagus vor, die ventrale, aufgebauschte, die Lungenanlage.

85. Hinter der Lungenanlage liegender Schnitt.

86. Leberanlage (Leb.).

Taf. VIII Fig. 1. Sagittalschnitt des Bodens der primitiven Pericardialhöhle eines Kaninchenembryo von $7_{1 \text { i }}$ mm Länge. Der hintere Theil des Embryos ist spiralförmig gewunden und daher ist sein Durchschnitt frontal, woraus sich dann die Lage der Massa longitudinalis (vgl. hierzu meine Arbeit über die Entwickelung des Zwerchfells, d. A. 22. Bd.) und der Vena umbil. erklärt.

Sin. v. $=$ sinus venosus

H. K. = Herzkammer.

Zott. = Zotten.

Ven. $=$ - Lebervenen, die ihren Urspruug direct aus dem Sinus venosus des Herzens nehmen.

Mes. = wuchernder Mesoblast.

$\mathrm{Leb}=$ Lebergewebe, in welchem die hypoblastischen und mesoblastischen Antheile nicht zu unterscheiden sind.

Bindg. = lockeres Bindegewebe (Hartn. 3, 4).

Fig. 2. Querschnitt des vordern Endes der Lungenanlage Nr. 81 der Taf. VII. (Hartn. 3, 7). Ausser der Erklärung zu Fig. 81 merke man:

Mes. = Mesoblast.

Bindg. $=$ Bindegewebe.

v. jug. = Ende der Vena jugularis sinistra. 